\title{
Novel Technique to Rescue a Folded Aortic Endograft during Endovascular Aneurysm Repair
}

\section{Jae Woong Lim}

Department of Thoracic and Cardiovascular Surgery, Soonchunhyang University Bucheon Hospital, Soonchunhyang University College of Medicine, Bucheon, Korea

Endovascular aneurysm repair has been established as a safe and effective treatment modality for infrarenal abdominal aortic aneurysms. Although rare, major technical failure can lead to intraoperative surgical conversion, which is associated with high mortality and morbidity rates. We herein report a case in which one of the endograft limbs was folded over on itself within the aneurysmal sac during endovascular aneurysm repair. The aneurysm was successfully repaired by a total endovascular approach using endoscopic grasping forceps without open surgical repair.

Keywords: Aorta; Aneurysm; Abdominal aorta; Complication

\section{INTRODUCTION}

Endovascular aneurysm repair (EVAR) has been proven safe and effective in the management of abdominal aortic aneurysms (AAAs) [1]; however, many complications related to endovascular procedures have been reported. Although acute conversion to open surgical repair is exceptional in current era, it is associated with high mortality and morbidity. We experienced a case in which the endograft limb was folded over on itself within the aneurysmal sac during EVAR and made limb catheterization impossible. The problem was resolved using endoscopic grasping forceps without open surgical repair. We successfully repaired the aneurysm by a total endovascular approach.

\section{CASE REPORT}

A 71-year-old man presented to Soonchunhyang University Bucheon Hospital with an AAA that had been incidentally detected by computed tomography. The maximal diameter of AAA was measured $7.6 \mathrm{~cm}$ with a proximal infrarenal neck diameter of 18 $\mathrm{mm}$ and length of $25 \mathrm{~mm}$. We planned to perform EVAR for the
AAA. In the operating room, both common femoral arteries (CFAs) were exposed under general anesthesia. An ${ }^{18} \mathrm{~F}$ sheath was introduced into each CFA, and the main body of the endograft ( $22 \mathrm{~mm}$; S\&G Biotech, Seoul, Korea) was placed in the proximal landing zone. Unfortunately, both renal arteries became blocked because of upward displacement of the main body after deployment. We attempted to move the main body inferiorly using a balloon catheter (33 mm, equalizer occlusion balloon; Boston Scientific, Natick, MA, USA) (Fig. 1A), but it was pulled down too far and fell down into the aneurysmal sac (Fig. 1B). The main body was fully distended within the aneurysmal sac, and we could not force it into the proximal landing zone. Thus, we sealed the graft using another thoracic stent graft $(24 \times 80 \mathrm{~mm})$ as a proximal cuff extending from the proximal landing zone to the main body (Fig. 2). After thoracic stent graft deployment, we could not cannulate the contralateral limb of the main body because it had folded when the main body fell into the aneurysmal sac. We tried to cannulate into the contralateral limb using cross-over technique and snaring technique from ipsilateral artery. Although using various types of wires and catheters, contralateral limb catheterization was failed.

Thus, we planned to unfold the limb with endoscopic grasping 


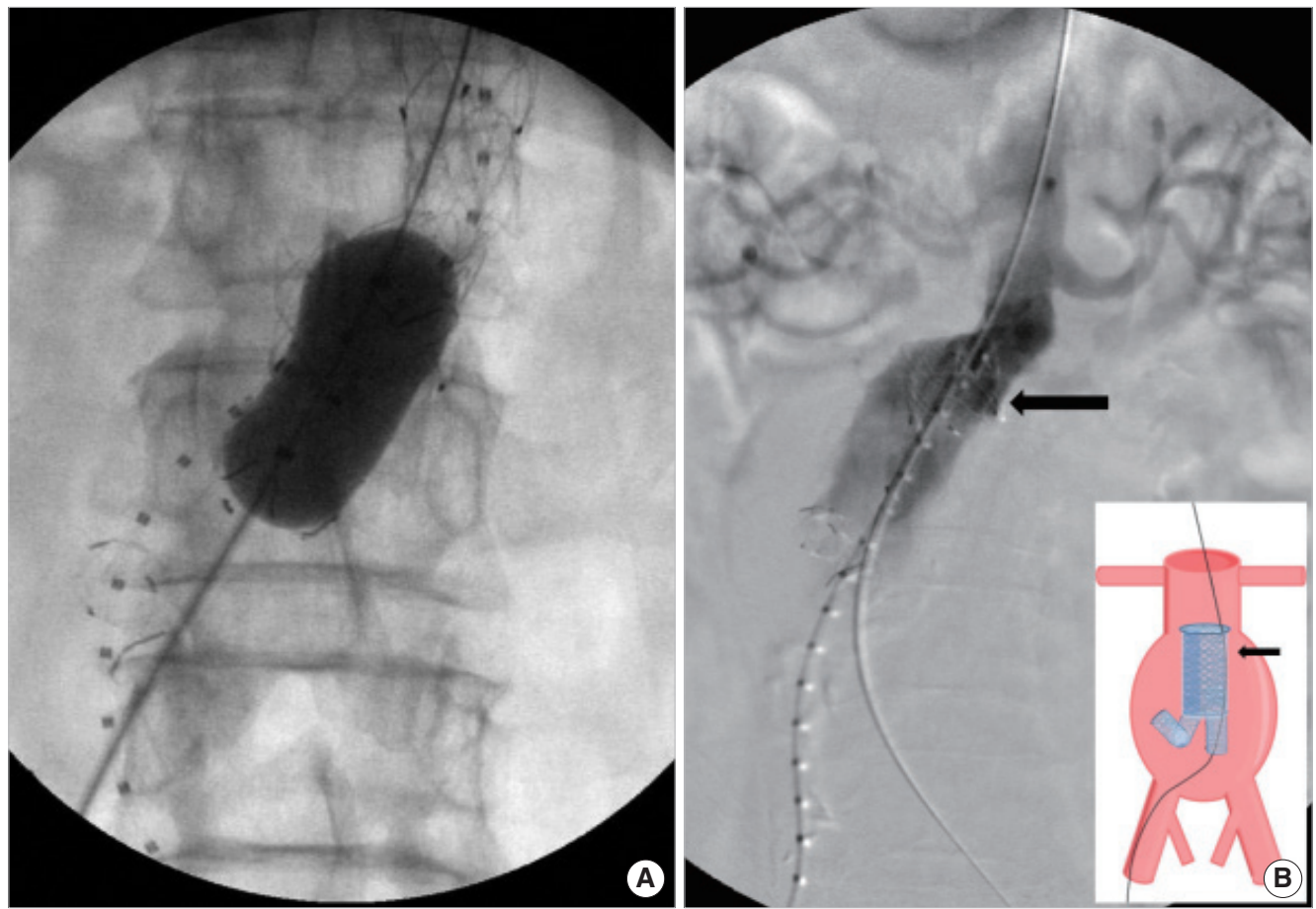

Fig. 1. (A) Intraoperative fluoroscopy showed a balloon to pull down the main body. (B) The black arrow indicates the main body fell into the aneurysmal sac.

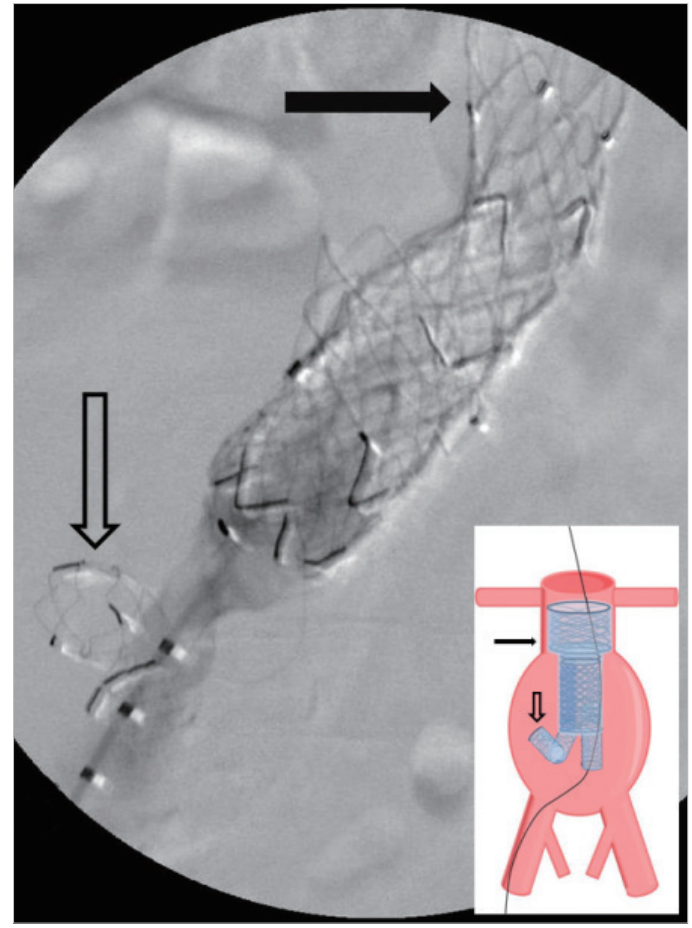

Fig. 2. Black arrow indicates thoracic stent graft $(24 \mathrm{~mm})$ and open arrow indicates the folded limb of main body.

forceps (model FG-32C-1; Olympus, Tokyo, Japan). A flexible, 2.8$\mathrm{mm}$ shark-tooth grasping forceps catheter, $105-\mathrm{cm}$ long, was in- troduced through the $18 \mathrm{~F}$ sheath of the right CFA. The distal portion of the kinked contralateral limb was visible due to the radiopaque makers. To minimize the risk of embolism or intimal injury, we carefully advanced the forceps to the kinked limb. We never opened the forceps before we identified that the forceps were exactly close to the kinked limb. We confirmed that the forceps were approached to the kinked limb under fluoroscopic guidance. The kinked limb was caught with forceps and pulled down into the aneurysmal sac. Thus, we unfolded the kinked limb and successfully cannulated the contralateral limb (Fig. 3). Finally, we inserted and deployed both limb stent grafts and finished the procedures (Fig. 3). The patient was discharged on postoperative day 12 and endoleaks or other complications were not observed on follow-up computed tomography (Fig. 4).

\section{DISCUSSION}

EVAR is associated with lower in-hospital mortality and major morbidity rates compared with open surgical repair [1,2], but this procedure is not risk-free. Although rare, major complications inevitably need acute intraoperative surgical conversion. According to several reports on open conversion after EVAR, early conversion may be necessary because of technical failure during deploy- 

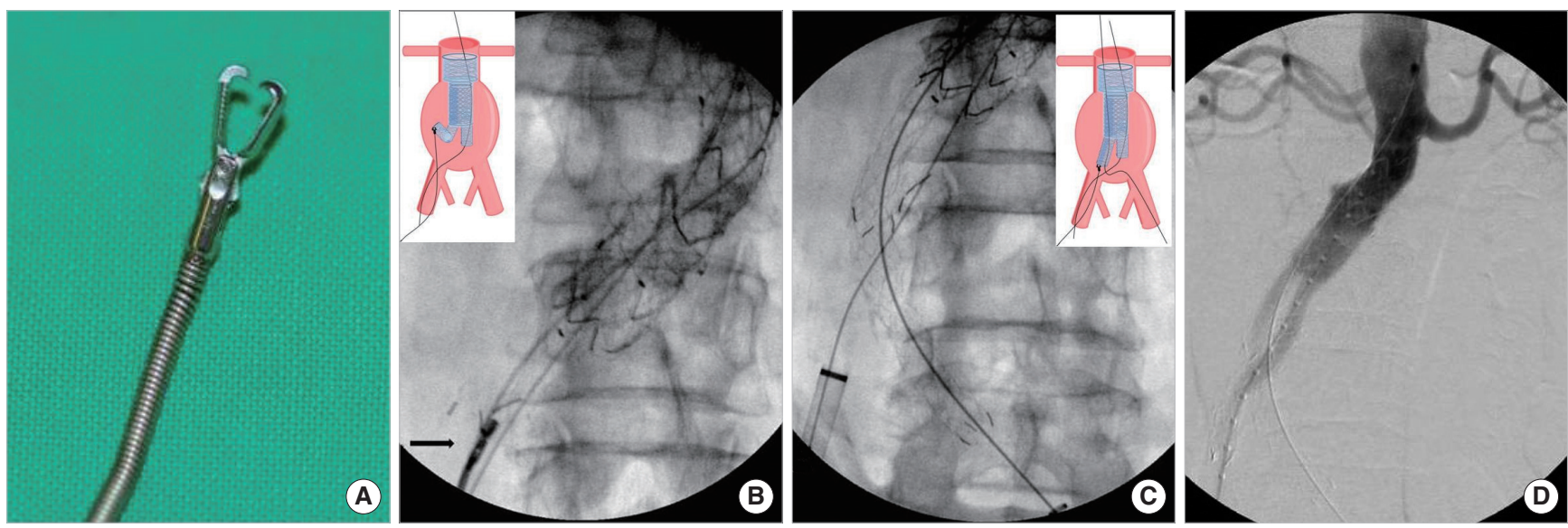

Fig. 3. (A) Endoscopic grasping forceps. (B, C) Fluoroscopy shows the unfolding of the main body limb and limb stent graft deployment. Black arrow indicates endoscopic grasping forceps pulling down the folded main body limb. (D) Final angiography shows the complete deployment of stent graft.

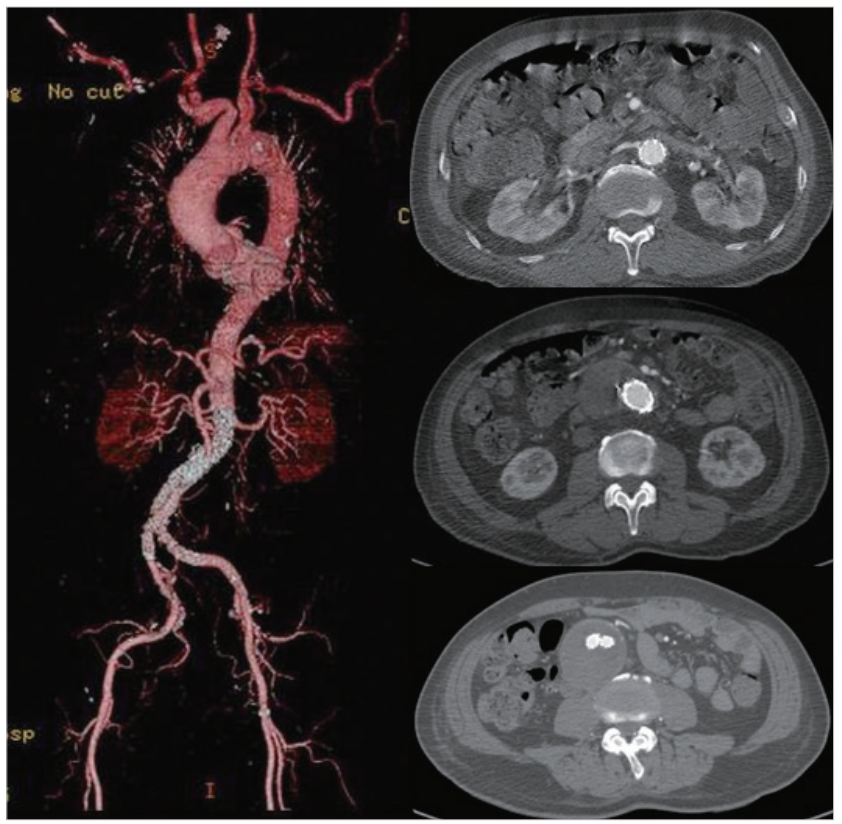

Fig. 4. Follow-up computed tomography angiography. The stent graft has maintained its shape and the endoleak is not observed.

ment, inappropriate stent graft placement, vessel rupture, or graft thrombosis. The most frequent cause of technical failure is severe calcification, stenosis, or tortuosity of the iliac arteries (primarily the external iliac artery), which may prohibit the passage of the device system up to the aorta [3,4]. Jacobowitz et al. [5] reported a $3 \%$ incidence of acute conversion and an $11 \%$ mortality rate, and showed that significant perioperative morbidity was common in patients with acute conversion. These series and other predominantly single-center experiences early in the evolution of EVAR demonstrated that acute conversion was an uncommon occur- rence but one that carried a significant attendant morbidity and mortality.

We encountered upward migration of the main body of the stent graft during deployment, resulting in the obstruction of both renal arteries. We attempted to move the main body inferiorly using the balloon catheter, but pulled it down too far so that it fell into the aneurysmal sac. This problem was resolved by the deployment of another thoracic stent graft precisely to the proximal landing zone. The main problem was that we could not cannulate the contralateral limb of the main body from the contralateral CFA because it had been folded when the main body fell into the aneurysmal sac. We attempted to cannulate the contralateral limb using all available different type catheters, but cannulation was unsuccessful. We had to performed laparotomy for removal of the stent graft and aneurysm repair. However, we tried to solve the problem with endovascular technique. The technique using endoscopic forceps may provide safe and efficacious treatment as a bailout procedure. Stavropoulos et al. [6] reported that rigid endobronchial forceps may be used as a reliable option for removal of embedded inferior vena cava filters. Many concerns about using of endoscopic forceps including embolic events, injury of aneurysmal wall should be considered. However appropriate anticoagulation and careful manipulation of endoscopic forceps would prevent these catastrophic complications.

We experienced a case in which the limb of the endograft folded over onto itself within the aneurysmal sac during EVAR due to technical error. However, using endoscopic grasping forceps, the aneurysm was successfully repaired by a total endovascular approach without open surgical repair. With this case, we would like 
Lim JW • Complication and Bail-out Procedure during Endovascular Aneurysm Repair

to highlight the utility of endoscopic grasping forceps not only for the removal of endovascular foreign body but also for the correction of endovascular stent graft instead of conversion to surgery.

\section{REFERENCES}

1. Greenhalgh RM, Brown LC, Kwong GP, Powell JT, Thompson SG; EVAR trial participants. Comparison of endovascular aneurysm repair with open repair in patients with abdominal aortic aneurysm (EVAR trial 1), 30-day operative mortality results: randomised controlled trial. Lancet 2004;364: 843-8.

2. Prinssen M, Verhoeven EL, Buth J, Cuypers PW, van Sambeek MR, Balm $\mathrm{R}$, et al. A randomized trial comparing conventional and endovascular repair of abdominal aortic aneurysms. N Engl J Med 2004;351:1607-18.

3. Jimenez JC, Moore WS, Quinones-Baldrich WJ. Acute and chronic open conversion after endovascular aortic aneurysm repair: a 14-year review. J Vasc Surg 2007;46:642-7.

4. Verzini F, Cao P, De Rango P, Parlani G, Xanthopoulos D, Iacono G, et al. Conversion to open repair after endografting for abdominal aortic aneurysm: causes, incidence and results. Eur J Vasc Endovasc Surg 2006;31: $136-42$.

5. Jacobowitz GR, Lee AM, Riles TS. Immediate and late explantation of endovascular aortic grafts: the endovascular technologies experience. J Vasc Surg 1999;29:309-16.

6. Stavropoulos SW, Dixon RG, Burke CT, Stavas JM, Shah A, ShlanskyGoldberg RD, et al. Embedded inferior vena cava filter removal: use of endobronchial forceps. J Vasc Interv Radiol 2008;19:1297-301. 